\title{
Whitening Processing of Seismic Signal Spectrum Based on SSGST
}

\author{
Shulin Zheng \\ School of Communication Engineering \\ Chengdu University of Information Technology \\ Sichuan, China
}

\begin{abstract}
In order to avoid the problem that single-domain spectral whitening cannot improve the time-frequency accuracy of seismic signals at the same time, this paper proposes a time-frequency domain spectral whitening based on synchrosqueezing generalized S transform(SSGST). First, the SSGST is used to perform time-frequency analysis on the seismic signal to obtain the time-frequency spectrum of the seismic signal. Then the whitening filter is used to perform spectral whitening on the timefrequency spectrum to obtain a seismic signal with higher time-frequency resolution. The verification of synthetic seismic signals and actual seismic signals proves that spectral whitening based on SSGST can simultaneously improve the time-frequency resolution of seismic signals, make the stratum information clearer, and have great significance for high-resolution processing of seismic signals.
\end{abstract}

Keywords: synchrosqueezing generalized S transform; spectral whitening; high resolution; seismic signal; data processing

\section{INTRODUCTION}

In seismic exploration technology, data interpretation occupies a very important position. Before interpreting seismic data, we must perform appropriate high-resolution processing on seismic data to further improve the accuracy of formation information estimation. Spectral whitening is one of the high-resolution processing methods. Its basic idea is to perform regional energy equalization of signals in the time domain or frequency domain to achieve the purpose of frequency compensation. In 1986, Bian Guozhu proposed spectral whitening of seismic data, and briefly described the principle of spectral whitening and its advantages in seismic signal processing ${ }^{[1]}$. In 2000, Chen Chuanren and others proposed to combine spectral whitening processing and wavelet transform. First, wavelet decomposition was used to decompose seismic signals to obtain signals of different scales, and then the signals were frequency compensated. Finally, the compensated signals were subjected to wavelet inverse transform, To obtain the high-resolution signal after spectral whitening ${ }^{[2]}$. In 2016, Lu Yiqi and others proposed to combine HHT with spectral whitening. First, use HilbertHuang transform to perform time-frequency analysis on seismic signals, and then whiten filter on time-frequency spectrum to obtain higher resolution seismic signals ${ }^{[3]}$. In 2018, Yan Zhonghui and others proposed to apply the Hilbert spectral whitening method to marine seismic data processing, which proved that the method is superior to conventional methods in local time-frequency characterization of signals and relative amplitude fidelity.

Based on reading related literatures, this paper proposes a point spectrum whitening based on synchrosqueezing generalized S transform (SSGST). Compared with Fourier transform, synchrosqueezing generalized $\mathrm{S}$ transform can process non-stationary signals; compared with wavelet transform, synchrosqueezing generalized $\mathrm{S}$ transform can obtain time frequency spectrum instead of time scale spectrum, which can more intuitively analyze seismic signals. Time domain and frequency domain. First, use SSGST to perform time-frequency analysis on the seismic signal to obtain the time-frequency spectrum of the seismic signal. Each point on the time-frequency spectrum has time and frequency characteristics. Then, using the whitening filter to whiten the time-frequency spectrum, we can obtain the high whitened spectrum. Resolution record.

\section{PRINCIPLE}

\subsection{SSGST}

The synchrosqueezing generalized $\mathrm{S}$ transform $(\mathrm{SSGST})^{[4]}$ is derived from the generalized $S$ transform ${ }^{[5]}$. It combines the advantages of the synchronous extrusion algorithm and the traditional GST algorithm. It can flexibly adjust the window function and adapt to various frequencies. Seismic signals can also make the time-frequency high-focus distribution and obtain a higher-resolution time-frequency spectrum map.

Assuming the original signal is $\mathrm{h}(t)$, its generalized $\mathrm{S}$ transform is expressed as:

$\int_{-\infty}^{+\infty} h(t) \frac{|f|^{r g s}}{\sqrt{2 \pi} \rho} \exp \left[-\frac{(\tau-t)^{2}|f|^{2 r g s}}{2 \rho^{2}}\right] \exp (-i 2 \pi f t) d t$

Where $\tau$ is the time change, $\mathrm{f}$ is the frequency, $\mathrm{rgs}$ and $\rho$ are the adjustment parameters of the window function. When rgs and $\rho$ are different, different window functions can be obtained, so that the generalized $\mathrm{S}$ transform is transformed into Gabor transform or standard $\mathrm{S}$ transform. According to the generalized $\mathrm{S}$ transform expression, we can derive the instantaneous frequency expression of the signal: $f_{h}(\tau, f)=\sum_{n=1}^{N}\left\{\delta\left(f-f_{n}\right)\left[f_{n}+\left(i 2 \pi G S T_{n}\left(\tau, f_{n}\right)\right)^{-1} \frac{\partial G S T_{n}\left(\tau, f_{n}\right)}{\partial \tau}\right]\right\}$

Where $\delta(f)$ is the impulse function, $f_{h}$ is the instantaneous frequency, and $f_{n}$ is the frequency.

According to the synchrosqueezing transform algorithm, we can get the spectrum of the original signal's synchrosqueezing generalized $\mathrm{S}$ transform: 


$$
\operatorname{SSGST}_{x}\left(f_{l}, \tau\right)=L_{f}^{-1} \sum_{f_{k}: f_{x}\left(f_{k}, \tau\right)-f_{i} \mid \leq \Delta f / 2} G S T(\tau, f) \exp \left(i 2 \pi f_{k} \tau\right) f_{k}^{-1} \Delta f_{k}
$$

Where $\tau$ is time variable, $f_{l}$ is the frequency of results, $f$ is frequency. $f_{k}$ is half the length of the frequency range. is the discrete frequency points.

\subsection{Spectral Whitening}

Different from the conventional time-domain spectral whitening or frequency-domain spectral whitening, in the time-frequency domain spectral whitening algorithm, we first need to perform time-frequency analysis on the original signal, and we design a whitening filter based on the obtained time-frequency spectrum.

$$
H\left(f, T_{i}\right)=\frac{\max \operatorname{SSGST}\left(f, T_{i}\right)}{\operatorname{SSGST}\left(f, T_{i}\right)+\varepsilon \max \operatorname{SSGST}\left(f, T_{i}\right)}
$$

Where the numerator is the maximum value of the amplitude spectrum at all times and is the white noise coefficient, $\varepsilon$ is used to adjust the signal-to-noise ratio and resolution of the signal after spectrum whitening. When it increases, the signal-to-noise ratio of the signal becomes larger, and the resolution decreases. When it decreases, the signal-to-noise ratio of the signal becomes smaller, and the resolution increases. Then, the generalized $\mathrm{S}$ inverse transform of the spectrum-whitened signal is simultaneously squeezed to obtain a high-resolution seismic record.

\section{APPLICATION}

We validate the above method using actual seismic data. Intercept the 1250th signal of 5000 actual seismic signals, and collect 2,000 sampling points per channel with a sampling frequency of $1 \mathrm{~ms}$.

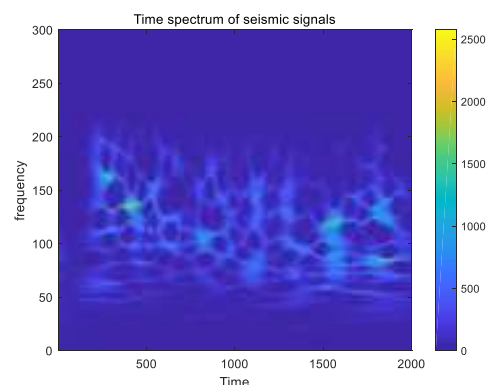

(a)

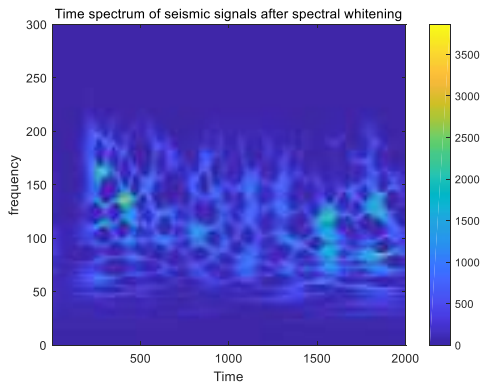

(b)

Figure1.Example of SSGST

Figure 1 (a) is the time-frequency diagram of the original single-channel seismic signal after SSGST processing. Figure. 1 (b) is a time-frequency spectrum diagram after spectrum whitening processing. By comparing (a) and (b), we can see that after spectral whitening, the amplitude of the signal divided in the high frequency range becomes larger, and the resolution is clearer. In the time-spectrum diagram, the detailed information of the signal after spectral whitening is supplemented, the width of the effective frequency band is increased, and the information of the high-frequency part is compensated, which can more effectively reflect the detailed information of the seismic signal.

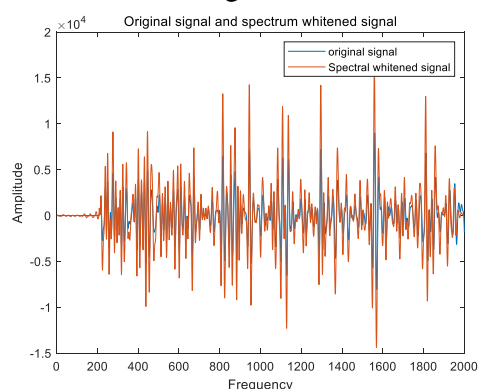

(a)

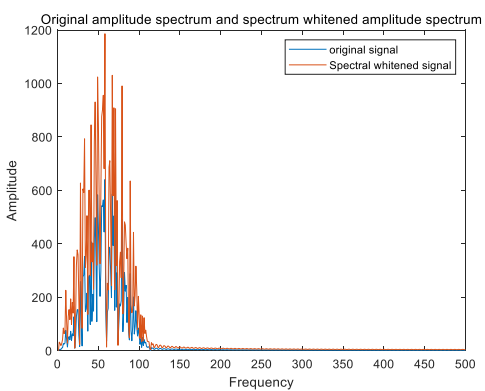

(b)

Figure2.example of Single-channel seismic signal

Figure 2 (a) is the comparison between the original signal and the spectrum-whitened signal. By performing ISSGST on the spectrum-whitened time-spectrum, we can reconstruct the original signal after spectrum-whitening. Figure 2 (b) is a comparison chart of the amplitude spectrum of the signal. It can be seen from the figure that the main frequency part of the seismic signal is compensated more and more detailed information is obtained.

Next, we perform spectral whitening on multiple seismic signals:

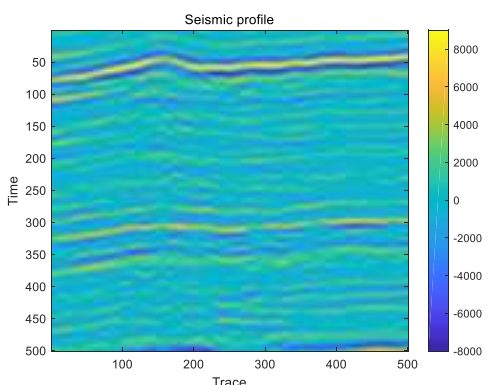

(a)

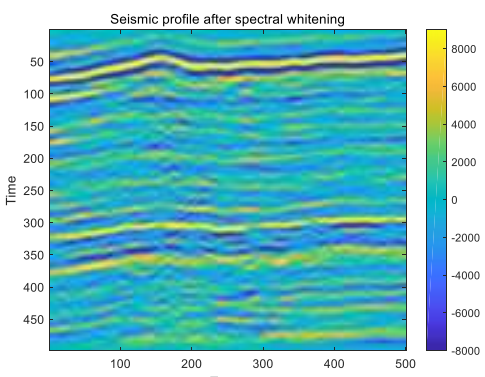

(b)

Figure3.Example of Multi-channel seismic signal

Figure 3 (a) is a cross-sectional view of the seismic signal. In the figure, 500 actual seismic signals are intercepted, and each 
channel collects 500 sampling points with a sampling frequency of 1 ms. Figure. 3 (b) is a seismic cross-sectional view after spectral whitening. Comparing the two pictures (a) and (b), it can be seen that the seismic profile after spectral whitening processing has more obvious layering information. The low-amplitude part of the high-frequency part in the original image is compensated and the resolution is improved. Stratigraphic conditions and their distribution are clearer.

\section{CONCLUSION}

The SSGST-based spectral whitening method proposed in this paper can effectively compensate seismic signals in the time and frequency domains. Time-frequency analysis is performed on the original signal, and then point spectrum whitening is performed on the time spectrum. Finally, ISSGST is performed on the spectrum-whitened signal to obtain a high-resolution seismic signal. This method overcomes the limitation that traditional spectral whitening methods can only improve time resolution or frequency resolution, compensates signals in the time-frequency domain, and has the characteristics that the SSGST algorithm can flexibly adjust the time window to adapt to different frequency signals. It is practical in high-resolution processing methods.

\section{REFERENCES}

[1] Guozhu, Bian. And Liqun, Zhang, J. 1986. Spectral Whitening of Seismic Data. Geophysical Prospecting for Petroleum.

[2] Chuanren, Chen.and Xirang, Zhou. J. 2000. Wavelet Spectrum Whitening Method Improves Resolution of Seismic Data. Petroleum Geophysical Exploration.

[3] Linzhi, Wu. And Hui, Chen. C. 2016. High Resolution Processing of Spectral Whitening Seismic Data Based on HHT Method. The 15th National Symposium on Mathematical Geology and Geoscience Information.

[4] Chen, H. And Lu, L. J. 2017. The Synchrosqueezing Algorithm Based on Generalized S-transform for HighPrecision Time-Frequency Analysis.

[5] Handing, H. And Na F. J. 2014. Research on Generalized S-Transform Seismic Resolution Method. Petroleum Geophysical Exploration 